\title{
A self-calibration rotational stitching method for precision measurement of revolving surfaces
}

\author{
M. Y. Liu, ${ }^{\mathrm{a},}$, C. F. Cheung ${ }^{\mathrm{a}}$, X. Feng ${ }^{\mathrm{b}}$, C. J. Wang, and R. K. Leach ${ }^{\mathrm{b}}$ \\ ${ }^{a}$ Partner State Key Laboratory of Ultra-precision Machining Technology, Department of Industrial and Systems \\ Engineering, The Hong Kong Polytechnic University, Kowloon, Hong Kong \\ ${ }^{\mathrm{b}}$ Manufacturing Metrology Team, Faculty of Engineering, University of Nottingham, Nottingham NG8 1BB, United \\ Kingdom \\ *corresponding author: samuel.liu@connect.polyu.hk
}

\begin{abstract}
When measuring revolving objects, it is often desired to obtain not only the geometrical form of the workpiece, but also the topography of the surface, as they both affect the performance of the part. However, holistic measurement of the entire three-dimensional surface of a revolving part is challenging since most surface measurement instruments only have limited measurement ability, where the bottom and the side surfaces cannot be measured. One solution to obtain geometrical form and surface topography information simultaneously is to add a precision axis to rotate the object while performing surface topography measurement. However, this solution requires a high-cost precision rotation stage and adjustable mounting and alignment aids. Moreover, errors in the rotation will be added to the measurement result, which can be difficult to compensate. Stitching is a method often used for measuring revolving surfaces without the need for precision motion axes, as the method is applied at the software level, and errors in the rotation can be compensated by the stitching algorithm. Nevertheless, the overall accuracy of stitching is limited when the number of sub-surfaces is large, since the measurement and stitching error accumulate along the stitching chain. In this paper, a self-calibration rotational stitching method is presented which can compensate for the accumulated error. The self-calibration method utilises the inherent nature of a revolving surface and compensates for the registration error by aligning the last dataset with the first dataset. The proposed method is demonstrated by measuring grinding wheels with a coherence scanning interferometer and simultaneously rotating the grinding wheels with a lowcost stepper-motor. It is demonstrated that the proposed stitching measurement method is effective in compensating for accumulated registration error. The proposed self-calibration rotational stitching method can be easily extended to a wide range of applications for measuring revolving surfaces using various measuring instruments.
\end{abstract}

Keywords: Rotational stitching, revolving surfaces, precision measurement, self-calibration, ultra-precision machining

\section{Introduction}

Revolving surfaces, such as precision rollers [1], shafts [2] and grinding wheels [3], are widely used in precision engineering. They are usually produced by a machining process that involves the rotational motion of spindles or motors, such as turning and grinding. There is an increasing demand for precision not only in the geometrical form of the machined part, but also in the surface texture, in applications such as producing microlens arrays on a roller stamper in order to replicate them onto a continuous flexible substrate [4] and optimising the microtopography on a diamond grinding wheel for improved grinding performance [5]. To holistically obtain both geometrical form and surface texture requires measuring the revolving surface with a surface measuring instrument and extracting both large-scale and small-scale information from the measurement. Measuring a revolving surface with a surface measuring instrument is a challenging task as most surface measuring instruments, such as coherence scanning interferometers (CSIs) and focus variation microscopes (FVMs), only have 2.5-dimensional (2.5D) measurement capability, where the bottom surface or the surface with high slope angle cannot be measured. In order to achieve three-dimensional (3D) measurement of the revolving surface, an additional precision rotational axis can be used to rotate the object, so that the entire surface can be measured. However, this method requires a high-cost precision rotational axis, and sometimes additional devices for tilting and alignment adjustment, which are not 
available to most surface measuring instruments, especially when large workpieces, such as precision roller drums in the roll-to-roll industry, are involved [6].

Measurement accuracy is often affected by the motion error of the rotational axis such as runout, angle error, misalignment between the workpiece and the rotational axis, and misalignment between the measuring probe and the rotational axis. The measurement error caused by the misalignment between the workpiece and the rotational axis is also subject to human errors and, therefore, cannot be easily controlled. One promising method to minimise this error is stitching, which utilises the common features in the overlapping region between two neighbouring fields of view to minimise alignment error. The stitching method is applied at the software level, independent of the instrument hardware setup. Several studies have focused on planar stitching, such as sub-aperture stitching interferometry for relatively flat surfaces [7, 8], and spherical and aspherical surfaces [9, 10]. Some studies have explored the measurement of revolving surfaces - specifically cylindrical surfaces. For example, stitching measurement of a full cylindrical surface has been studied by Peng et al. [11, 12], with a method based on a Fizeau type interferometer and a computer generated hologram (CGH) null. The method achieves sub-micron accuracy, but it was only applicable to high precision cylindrical surfaces with a specific diameter, since different CGH nulls would be needed when the size of the cylinder changes. Weckenmann et al. [13] developed grazing incidence interferometry for high-precision measurement of cylindrical form deviations. The method utilised the incident laser beam to project to the measured cylindrical surface and the interferogram was recorded by the CCD camera. The interferogram could then be used to determine the cylindrical form deviations. Guo and Chen [14] developed a multi-view connection technique for $360^{\circ}$ 3D measurement using fringe projection, where the object was placed on a precision rotary stage and two sets of fringe patterns were projected onto the overlapped regions at different angles. The measured topographies were then stitched together using an iterative least-squares algorithm. However, the accuracy of the method was not determined. Vissiere et al. [15] developed a cylinder measuring machine with a dissociated metrology technique (DMT)-based architecture using a modified multi-step error separation method to eliminate the spindle errors. To achieve the ultimate $5 \mathrm{~nm}$ measurement uncertainty level, the machine was designed with sophisticated hardware. Neugebauer et al. [16] developed a comparator for the measurement of diameter and form of cylinders, spheres and cubes. With a twoprobing system adhering to the Abbe principle and a form reference ring, the measurement uncertainty was as low as $20 \mathrm{~nm}$. Most of the above mentioned revolving measurement instruments were specially designed machines dedicated for measuring specific types of revolving surfaces but not for generic revolving surfaces. Most of these instruments also involved sophisticated hardware and are not widely available in industry.

This paper presents a self-calibration rotational stitching (SCRS) method for precision measurement of generic revolving surfaces that can be applied to any surface topography measuring instrument. The proposed SCRS method utilises a traditional 2.5D surface topography measurement instrument, such as CSI. Instead of using a highprecision rotational axis, this method only requires a low-cost rotating motor, such as a stepper motor for rough positioning of the measured revolving surface. Measurement of the entire revolving surface is divided into multiple sub-surfaces with overlapped regions; the sub-surfaces are stitched together initially using the surface registration method. The accumulated measurement and registration error is minimised by the SCRS method which makes use of the first and the last sub-surface in the stitching loop. The proposed method is presented in detail, demonstrated by measuring precision diamond grinding wheels, and is shown to be effective. Due to its generality, the proposed method can be applied to measure various types of revolving surfaces such as cones, spheres and cylinders. The SCRS method can be easily implemented to existing 2.5D surface measuring instruments to enable true 3D measurement of revolving surfaces. In addition, a software user interface (UI) is developed to synchronise rotation of the object with measurement, without the need for an application program interface (API) to the instrument software, making the proposed method truly independent of measuring instruments.

\section{Self-calibration rotational stitching (SCRS) method}

The schematic diagram of the proposed SCRS method for measuring revolving surfaces is shown in Figure 1. The method is demonstrated on a CSI with a rotational axis driven by a stepper motor. The SCRS method is divided into two steps. The first step is data acquisition of the revolving surface topography by synchronised measurement while the sample is being rotated. For every measurement, only a small part on the top of the revolving surface is measured because of the limited field of view (FoV). The number of required sub-surfaces is determined by the FoV 
of the measuring instrument, the dimension of the revolving surface and the percentage of overlapped region. After each measurement, the stepper motor rotates by a specific angle so that the sub-surfaces are overlapped by the required percentage of region for registration purposes. Automatic measurement is implemented using purposely designed software running on the personal computer (PC) that controls the CSI. Since the topographical accuracy of the measurement is ensured by the CSI and the registration process, the motion error of the rotational axis and the misalignment between the revolving surface and the rotational axis are minimised using the proposed SCRS method, which is described in the following section.

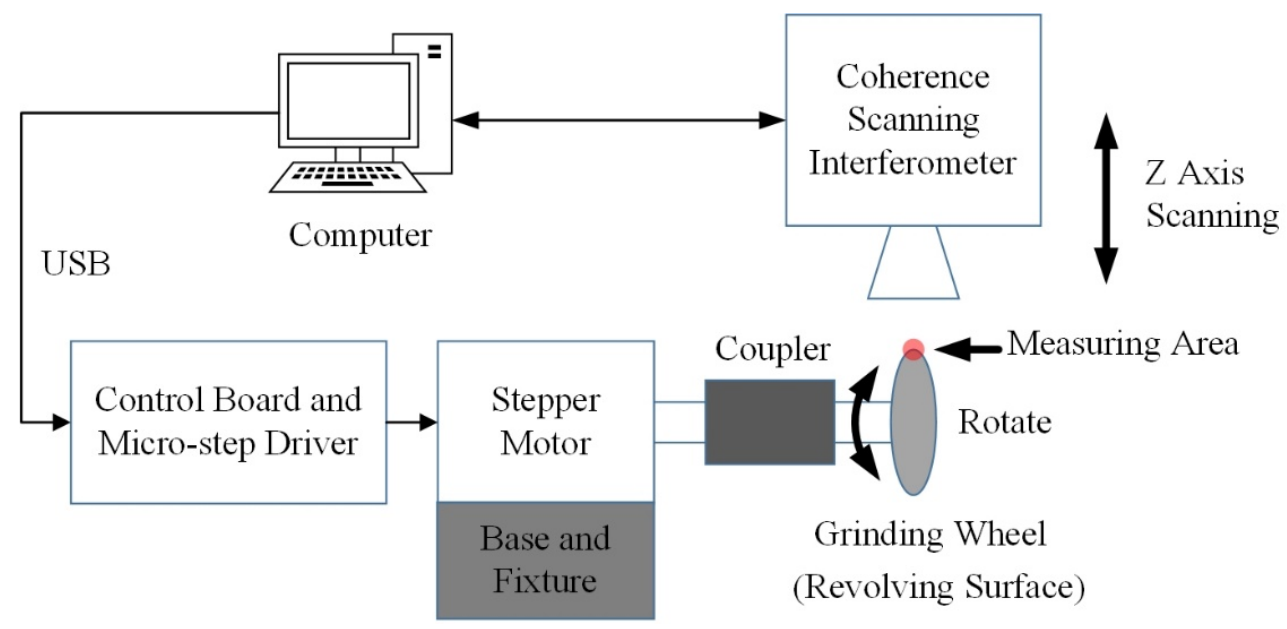

Figure 1 Schematic diagram of rotational stitching measurement of revolving surfaces.

The second step is the stitching of acquired individual topographies. A flow chart of the stitching algorithm is shown in Figure 2. After pre-processing of the original dataset, such as removing null data and outliers, the registration process is performed on every pair of neighbouring datasets. The subsequent dataset is registered onto the previous dataset, and the transformation matrix of the subsequent dataset is computed. With a priori knowledge of the radius of the revolving surface and the rotating angle for every individual measurement, the overlapped regions between neighbouring datasets can be roughly determined, and an initial alignment can be performed. The initial transformation serves as rough registration to address the local minima issue, where the registration process is trapped in a local, albeit incorrect, zone with minimum root mean square error. The roughly registered datasets are then fine registered using the iterative closest point (ICP) algorithm [17]. The ICP algorithm is a numerical method which iteratively calculates the transformation matrix $(4 \times 4)$ for the measured surface with the aim that the transformed surface (points) is closest to the reference surface (points). Due to the fact that the measurement uncertainty of the CSI is affected by the local surface slope $[18,19]$, an adaptive registration approach is proposed to improve the accuracy of the fine registration process, which automatically and adaptively selects points with low measurement uncertainty for registration. The adaptive registration approach generates an error map of each registration, and points with large registration errors due to large local surface slopes are removed. Registration is further iterated until the registration error is smaller than a pre-defined threshold. With the adaptive registration approach, points with high measurement uncertainty are excluded automatically, which is essential when dealing with a large number of datasets (e.g. 200 datasets required for the grinding wheel).

After fine registration of all the neighbouring datasets, the transformation matrices of every dataset are determined, and the entire revolving surface can be reconstructed. It is interesting to note that, the final stitching error can often be large when dealing with a large number of sub-surfaces since measurement errors and registration errors are accumulated along the stitching process. As a result, the last dataset is expected to have the largest accumulated misalignment error, while in the ideal situation (without measurement error and registration error), the error should be zero. Therefore, we propose a self-calibration method to minimise the accumulated error by using the first dataset as the wrap-around sub-surface. A fine-tuned transformation matrix is applied to every dataset (except for the first one) so as to implement the self-calibration process. The optimum transformation matrix is found where the last dataset aligns well to the first dataset to form a close loop of the revolving surface. As a result, all the datasets are well 
registered to neighbouring datasets in the whole loop. After the initial transformation, the matrices for registration and the fine-tuned transformation matrices are combined to transform all the original datasets into a stitched topography of the revolving surface.

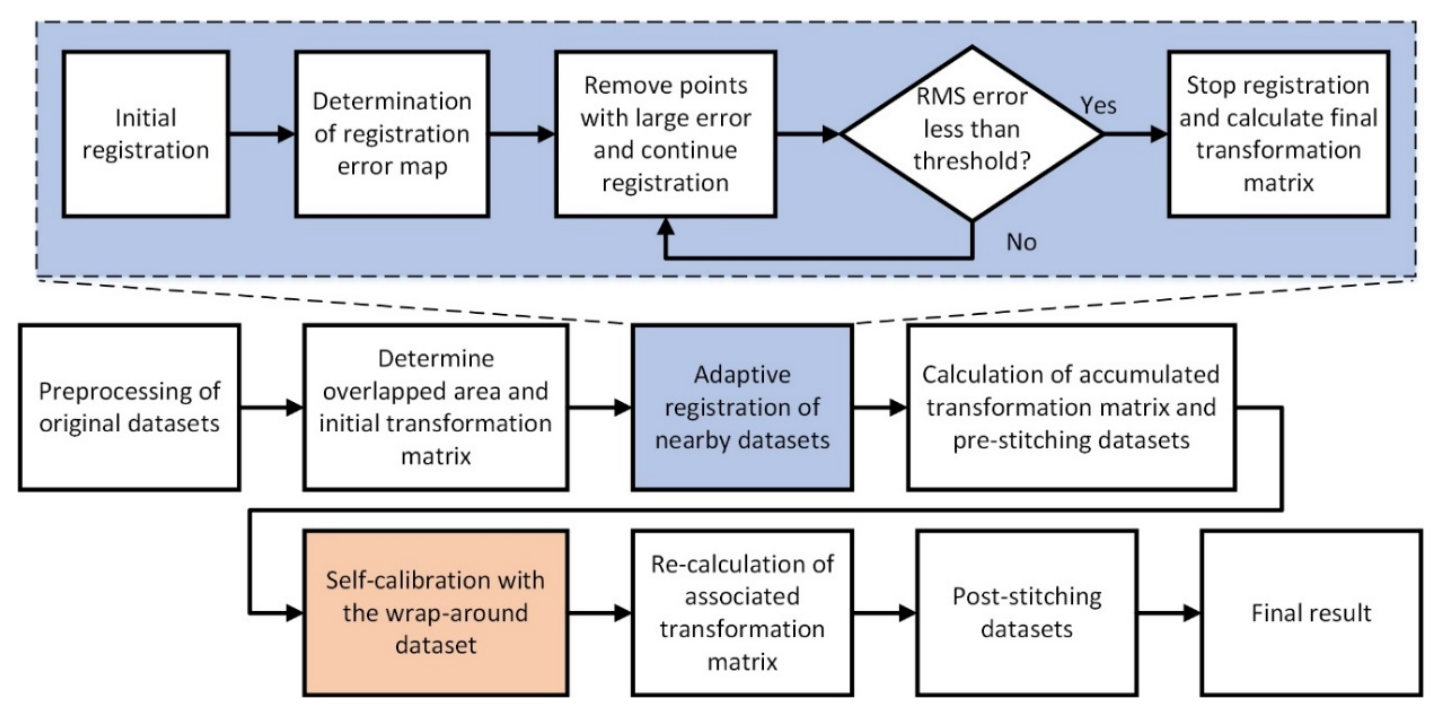

Figure 2 Flow chart of the rotational stitching measurement with adaptive registration and self-calibration method.

\subsection{Hardware and software setup for data acquisition}

Although the proposed method is implemented at the software level, special attention should also be paid to the hardware setup to avoid unnecessarily large errors during data acquisition. Since the vertical scanning range of the measuring instrument is often limited (e.g. $150 \mu \mathrm{m}$ for the CSI used in the experiment), the runout of the revolving surface should be minimised to have large and evenly distributed measurable area of every dataset, thus avoiding large unmeasurable areas. This is implemented by improving the coaxiality between the workpiece and the motor shaft as shown in Figure 1. The magnification of the objective lens is selected according to the required lateral resolution. The minimum number of required sub-surfaces can then be determined and the incremental angle of object rotation for each measurement is calculated, which aims to ensure an optimum $20 \%$ overlapped region between sub-surfaces [20]. Hence, the number of sub-surfaces $N_{S}$ and the rotation angle $A_{R}$ can be determined by

$$
\begin{aligned}
& N_{S} \geq \frac{(1+r) P}{L} \\
& A_{R} \leq \frac{L}{(1+r) P} \times 360^{\circ}
\end{aligned}
$$

where $r$ is the overlap ratio with an optimum value of $20 \%, P$ is the estimated perimeter of the revolving object, and $L$ is the measurement range of the sub-surface in the perimeter direction, which is mainly determined by the FoV. It should be noted that a larger overlapped region can improve stitching accuracy, but will result in longer measurement duration.

After the objective magnification and the incremental rotation angle are determined, the measurement process can be started. It is necessary to automate the measurement process as often a large number of measurements (e.g. 200 measurements for the grinding wheel involved in this study) are required. Since there is no API for the CSI to communicate with the stepper motor, which is the case with most commercial measuring instruments, an interface was developed to synchronise the rotation of the object while the CSI is set to repeatedly measure for $N_{s}$ times. The interface software monitors a folder where CSI measurement data is stored. Once a new data file is detected, indicating 
completion of a measurement, a command is sent to drive the stepper motor by a pre-set angle. The command is sent through USB cable to the control board of the stepper motors. With this method, the data acquisition process is completed automatically without the need to interface with the software of the CSI.

\subsection{Pre-processing original datasets and initial registration}

The original datasets obtained by the CSI contain a significant number of non-measurable null data, which is likely caused by the large local slope of the rough surface or optical noise [21]. These null data points need to be removed first. After that, in each pair of neighbouring datasets, the latter one is roughly aligned to the former, with the initial transformation derived by the a priori information of the measurement setup. The homogeneous transformation matrix [22] of the initial transformation is determined by translations along $x, y, z$ axes and rotations about $x, y, z$ axes. The translations can be determined by

$$
T\left(v_{x}, v_{y}, v_{z}\right)=\left[\begin{array}{cccc}
1 & 0 & 0 & v_{x} \\
0 & 1 & 0 & v_{y} \\
0 & 0 & 1 & v_{z} \\
0 & 0 & 0 & 1
\end{array}\right]
$$

where $v_{x}, v_{y}, v_{z}$ are the translation distances along $x, y, z$ axes, respectively. Rotations about $x, y, z$ axes can be determined by

$$
\begin{aligned}
& R_{x}(\alpha)=\left[\begin{array}{cccc}
1 & 0 & 0 & 0 \\
0 & \cos (\alpha) & -\sin (\alpha) & 0 \\
0 & \sin (\alpha) & \cos (\alpha) & 0 \\
0 & 0 & 0 & 1
\end{array}\right] \\
& R_{y}(\beta)=\left[\begin{array}{cccc}
\cos (\beta) & 0 & \sin (\beta) & 0 \\
0 & 1 & 0 & 0 \\
-\sin (\beta) & 0 & \cos (\beta) & 0 \\
0 & 0 & 0 & 1
\end{array}\right] \\
& R_{z}(\gamma)=\left[\begin{array}{cccc}
\cos (\gamma) & -\sin (\gamma) & 0 & 0 \\
\sin (\gamma) & \cos (\gamma) & 0 & 0 \\
0 & 0 & 1 & 0 \\
0 & 0 & 0 & 1
\end{array}\right]
\end{aligned}
$$

where $\alpha, \beta$, and $\gamma$ are the rotation angles about $x, y, z$ axes, respectively.

The initial transformation $T_{I}$ in rough alignment is determined by the a priori knowledge of the radius of the grinding wheel and the rotation angle $A_{R}$ for each step. The initial registration is a rough registration process which aims to improve the stability of the subsequent fine registration process, which is susceptible to local minima issues when the ICP algorithm [17] is used.

\subsection{Adaptive fine registration with ICP algorithm}

Ideally, the results of two measurements at the same area are identical. However, due to the fact that the surface topography of every single measurement of the revolving surface may contain areas of high surface slope, registration error will be introduced due to higher measurement uncertainty in those areas $[18,19]$. To address this issue and improve the registration accuracy, we propose an adaptive fine registration process based on the ICP method [17], which is undertaken as follows: 
Step (a): The ICP registration is implemented. Transformation matrix $T_{I C P}$ is used to transform the dataset and the error map is obtained.

Step (b): Points with large registration errors in the transformed dataset are identified and removed to form a new dataset.

Step (c) goes to step (a) and continues the registration process with the new dataset until the registration error is less than the predefined threshold.

After the adaptive fine registration is completed, a series of transformation matrices are determined for each pair of datasets and are denoted as $T_{I C P 1}, T_{I C P 2}, \ldots, T_{I C P m}$, where $m$ is the number of iterations in the ICP registration process. Subsequently, the final transformation matrix $T_{\text {ICPF }}$ can be determined as

$$
T_{I C P F}=T_{I C P m} T_{I C P(m-1)} \cdots T_{I C P 2} T_{I C P 1}
$$

where all the transformation matrixes are $4 \times 4$ homogeneous matrices. The adaptive registration process automatically excludes outliers and noisy data (e.g. due to large surface roughness and high surface slope angle) in the topographies during the registration process, hence improving registration accuracy. The process is iterated until registration error is below a predefined threshold.

\subsection{Calculation of accumulated transformation matrix and pre-stitching datasets}

After the initial and fine transformations for neighbouring datasets are determined, the accumulated transformation matrix for each dataset can be determined. In this study, the coordinates of the first measurement dataset remain unchanged during the process, while the other datasets are transformed into the same coordinate system. The accumulated transformation matrix for the 2 nd to the $n$th dataset, $T_{A i}$, is determined as

$$
T_{A i}=\prod_{j=2}^{i}\left(T_{\text {ICPFj }} T_{I}\right)
$$

where $i=2 \mathrm{~L} n, T_{\text {ICPFj }}$ is the $j$ th fine transformation matrix $T_{\text {ICPF }}$ for the $j$ th dataset to the $(j-1)$ th dataset, where $j=2 \mathrm{~L} i \cdot T_{I}$ is the initial rough transformation matrix for the $j$ th dataset to the $(j-1)$ th dataset and it has the same value for each pair of dataset.

After the accumulated transformation matrices for each dataset are determined, all the original datasets can be stitched together to form a holistic dataset.

\subsection{Self-calibration method using the wrap-around dataset}

The accumulated stitching error is determined by calculating the misalignment between the first dataset and the last dataset, and the misalignment error is determined as follows. The first dataset is duplicated and added to the end of the loop as the $(n+1)$ th dataset, using the same rough and fine registration method. In the ideal situation without measurement and registration error, the transformed $(n+1)$ th dataset should be identical to the first dataset. In practice, misalignment between the $(n+1)$ th dataset and the first dataset will always be present. It is reasonable to assume that with the same measurement setup and registration method, the incremental misalignment error for each pair of datasets are approximately the same. With that assumption, the constant misalignment error can be determined and compensated for by introducing an associated transformation matrix $T_{C}$ to minimise the misalignment between the first dataset and the $(n+1)$ th dataset. The associated transformation matrix $T_{C}$ is applied to each pair of datasets. The misalignment error should have 6 degrees-of-freedoms (DOFs) and the associated transformation matrix $T_{C}$ to compensate for this error is determined by

$$
T_{A(n+1)}=\prod_{j=2}^{n+1}\left(T_{C} T_{I C P F j} T_{I}\right)=I
$$


where $T_{I C P F(n+1)}$ is the transform matrix for the fine registration for the first dataset to the last dataset (the $n$th one) and

$I$ is the identity matrix which denotes all the translation components and the rotation components in the transformation matrix are zero. Since $T_{I C P F j}$ and $T_{I}$ are known, $T_{C}$ can be determined. It should be noted that $T_{I C P F j}, T_{I}$ and $T_{C}$ are all $4 \times 4$ homogeneous transformation matrices. Solving $T_{C}$ is a classical non-linear problem and is determined by using the Levenberg-Marquardt algorithm (LMA) [23]. The calculation of the misalignment error is determined by using the dataset itself, and hence, it is a self-calibration method.

\subsection{Finalisation of the transformation matrix for each dataset}

After the self-calibration calculation, the final transformation matrix for each dataset can be determined by

$$
T_{F i}=\prod_{j=2}^{i}\left(T_{C} T_{\text {ICPFj }} T_{I}\right)
$$

where $i=2 \mathrm{~L} n, T_{C}$ is the compensation transformation matrix calculated by using the self-calibration method, $T_{I C P F j}$ is the $j$ th fine transformation matrix $T_{I C P F}$ for the $j$ th dataset to the $(j-1)$ th dataset, where $j=2 \mathrm{~L} i . T_{I}$ is the initial transformation matrix for the $j$ th dataset to the $(j-1)$ th dataset, and has the same value for each pair of dataset. After the transformation matrices are finalised, the complete revolving surface can be reconstructed.

\section{Experiments and discussions}

To demonstrate the effectiveness of the proposed method, an experiment was conducted on the revolving surface of a grinding wheel. The measuring instrument used in the experiment was ZYGO Nexview CSI with 20× objective lens and $1 \times$ zoom lens, resulting in a lateral resolution of $0.4 \mu \mathrm{m}$ (pixel spacing). A diamond grinding wheel from DIAGRIND INC with a nominal diameter of $14 \mathrm{~mm}$ was used. The surface roughness $\left(S_{a}\right)$ of the grinding wheel is approximately $1.8 \mu \mathrm{m}$. A SANYO DENKI $35 \mathrm{~mm}$ stepper motor with $1.8^{\circ} / \mathrm{step}$ was used while the controller was an Arduino UNO and the micro-stepping driver was M415B from Leadshine Technology Co. Ltd. The static angle error of the motor is $\pm 0.09^{\circ}$ and the shaft runout is $0.025 \mathrm{~mm}$. Larger angle error is acceptable as it mainly causes lateral misalignment, which can be corrected by stitching. Shaft runout causes the height of the measured regions to vary during rotation. Larger runout will require the measuring instrument to increase the vertical scanning range, thus increasing measurement period significantly. For the grinding wheel measured in this experiment, $50 \%$ is the smallest overlapped area achievable with a single step motion $\left(1.8^{\circ}\right)$ by the stepper motor. Given the $14 \mathrm{~mm}$ diameter of the grinding wheel, $3.6^{\circ}$ rotation angle would have resulted in $0 \%$ overlapped region, making it impossible to perform stitching. Hence, $200\left(360^{\circ} / 1.8^{\circ}\right)$ datasets were required to cover the entire surface. The vertical scanning range of the CSI was set to be $150 \mu \mathrm{m}$ (maximum) so as to obtain as much data as possible given the runout of the shaft. Figure 3 shows the experimental setup of the measurement process. Figure 4 shows the UI of the CSI software, the UI of the self-developed control software and the folder containing measured data files. The 200 measurements performed to measure the entire revolving surface lasted for 2 hours.

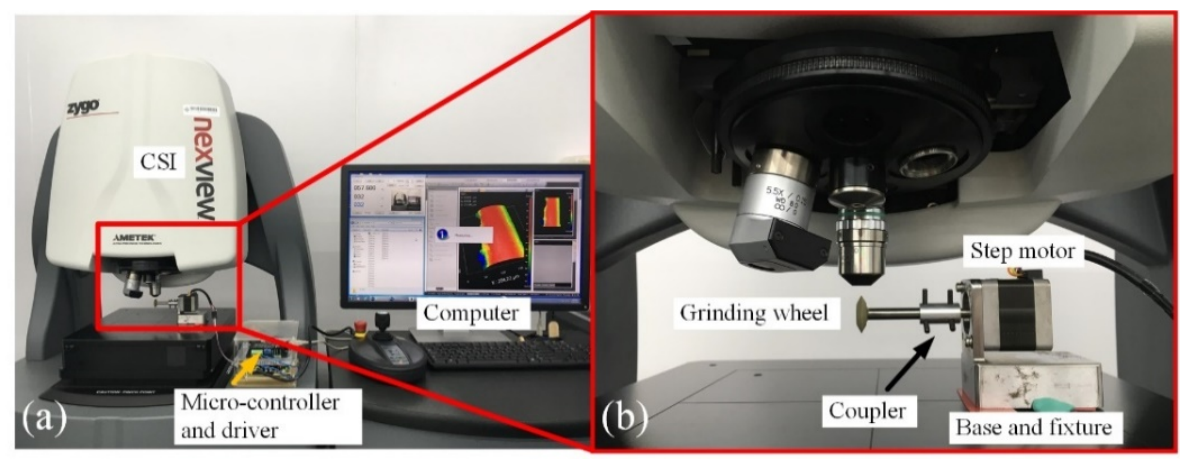

Figure 3 Measurement experiment setup, (a) the setup showing the CSI, the UI of the CSI and the grinding wheel,

(b) zoom-in view for the grinding wheel. 


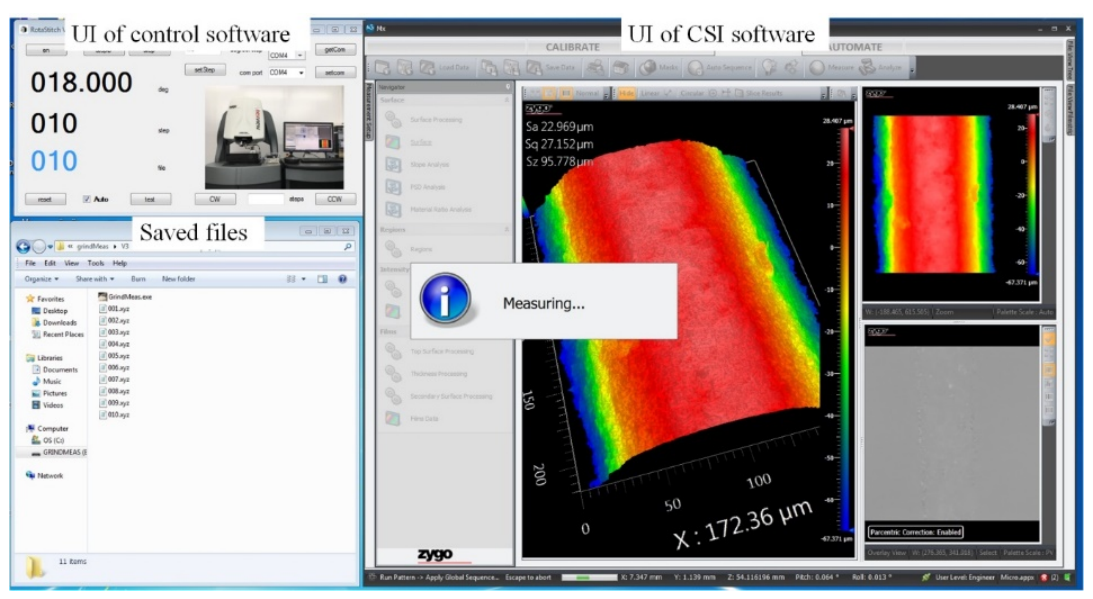

Figure 4 Captured screen showing the UI of the CSI software, UI of the control software and the measured data files.

Some of the original datasets are shown in Figure 5. They show that there is a large overlapped region (about $50 \%$ ) between neighbouring datasets which is used to ensure registration accuracy. The presence of unique nonrepetitive micro-topography patterns on the grinding wheel is observed, which benefit the registration process.

(a)

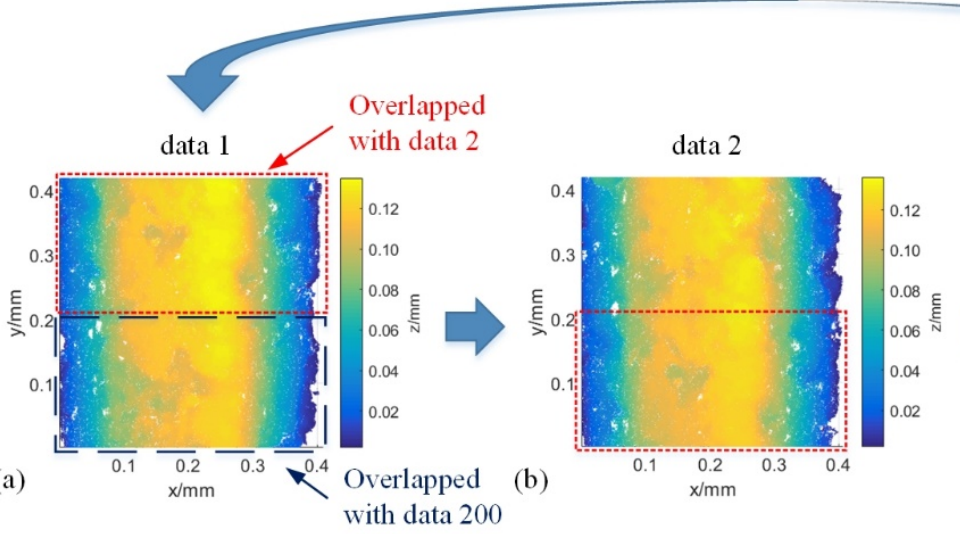

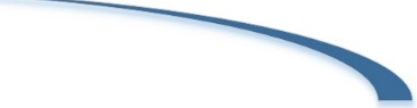

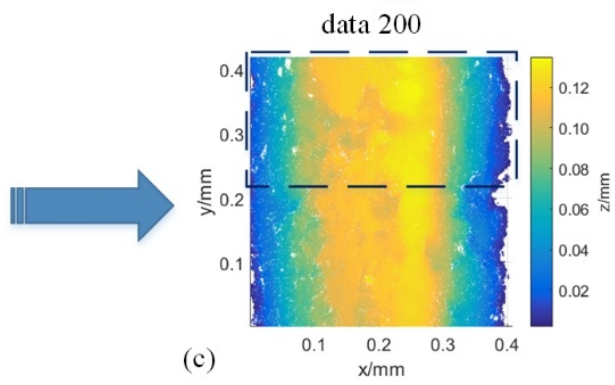

Figure 5 Original datasets, (a) data 1 with marked area overlapped with data 2 and data 200, (b) data 2 with marked area overlapped with data 1, (c) data 200 with marked area overlapped with data 1.

During rough registration, it was found that translation along the perimeter direction alone was sufficient to successfully avoid the local minima issue. The initial transformation matrix $T_{I}$ was, therefore, determined to be:

$$
T_{I}=\left[\begin{array}{cccc}
1 & 0 & 0 & 0 \\
0 & 1 & 0 & T_{y} \\
0 & 0 & 1 & 0 \\
0 & 0 & 0 & 1
\end{array}\right]
$$

where $T_{y}=0.22 \mathrm{~mm}$ and it is determined by

$$
T_{y}=R \beta
$$

where $R=7 \mathrm{~mm}$ is the nominal radius of the wheel, and $\beta=1.8 \pi / 180 \approx 0.0314$ is the incremental rotation angle of the stepper motor in radians. 
An example of adaptive registration between the first dataset and the second dataset is shown in Figure 6 . The adaptive registration was implemented in 4 iterations of ICP registration; after each iteration data points outside 0.2 times the root-mean-squared (RMS) registration error was removed from the second dataset. Within each iteration of ICP registration, the ICP algorithm was set to iterate 100 times. As points with large errors were iteratively excluded from the calculation, the RMS errors after every registration are $2.0139 \times 10^{-4} \mathrm{~mm}, 2.2875 \times 10^{-5} \mathrm{~mm}, 3.6144 \times 10^{-6} \mathrm{~mm}$ and $2.8813 \times 10^{-6} \mathrm{~mm}$, respectively, showing a gradual decrease. The number of points in the second dataset included in the registration process after each iteration are $3.5 \times 10^{5}, 3.2 \times 10^{5}, 5.6 \times 10^{4}$ and $6.6 \times 10^{3}$, respectively.
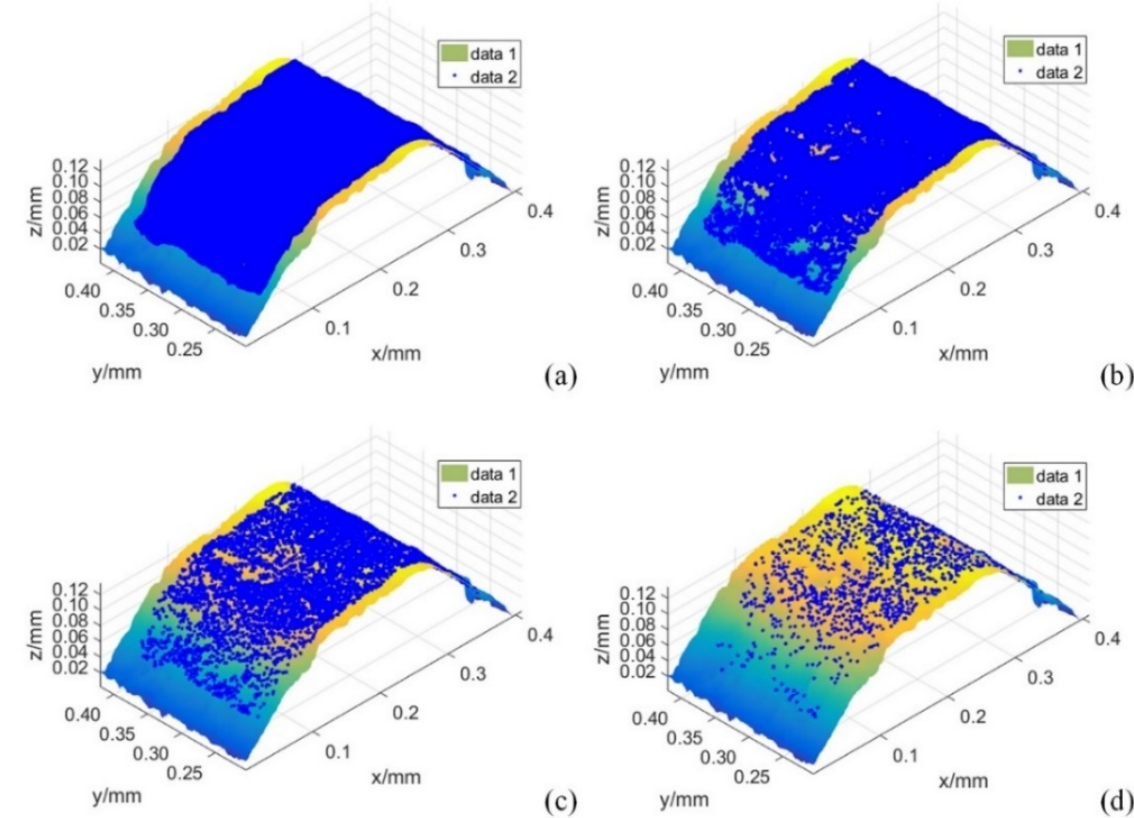

Figure 6 Adaptive registration between the first and second dataset, (a) after first iteration, (b) after second iteration, (c) after third iteration, and (d) after fourth iteration.

The RMS of the registration errors for all 200 datasets during adaptive registration are shown in Figure 7. It can be observed that a few spikes are present during the first three iterations, indicating large registration errors between specific pairs of datasets. These large errors are due to the inclusion of data points with large local surface gradient, which were successfully removed after the fourth iteration. Figure 8 shows the pair of datasets 133 and 134 that correspond to the highest spike in Figure 7. After removing the data points causing the large registration error iteratively, the registration error in the last iteration is much smaller and evenly distributed along all the datasets. 

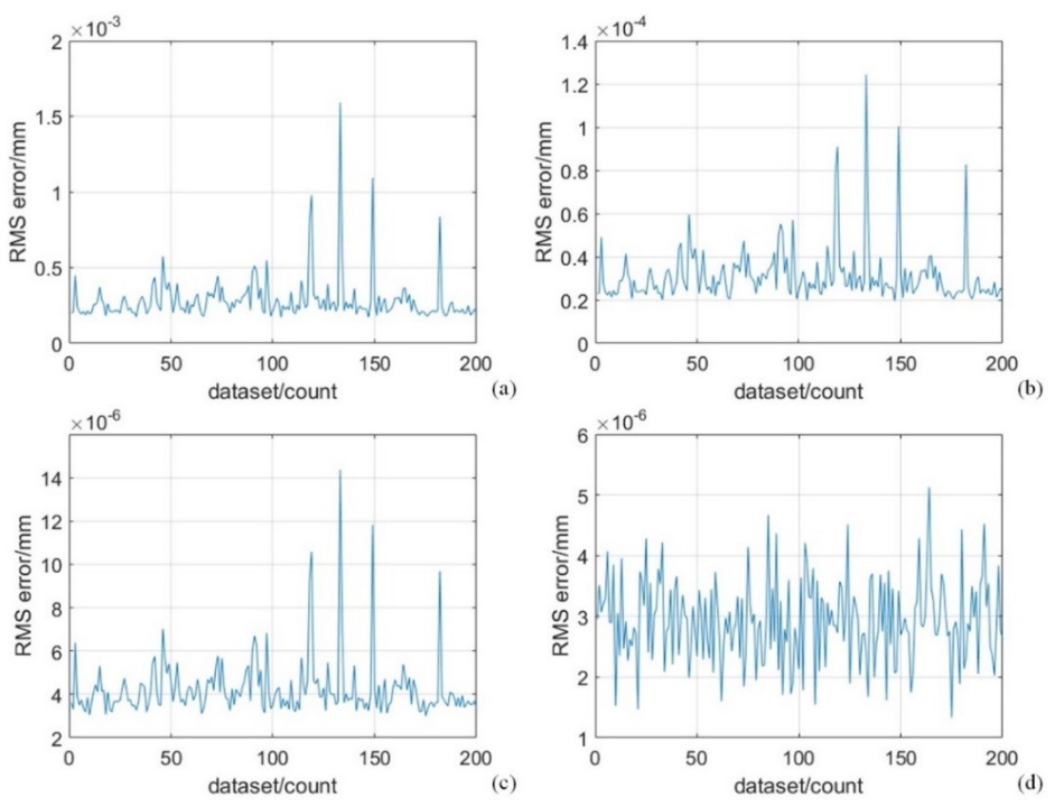

Figure 7 RMS values of the registration error, (a) after first iteration, (b) after second iteration, (c) after third iteration, and (d) after forth iteration.

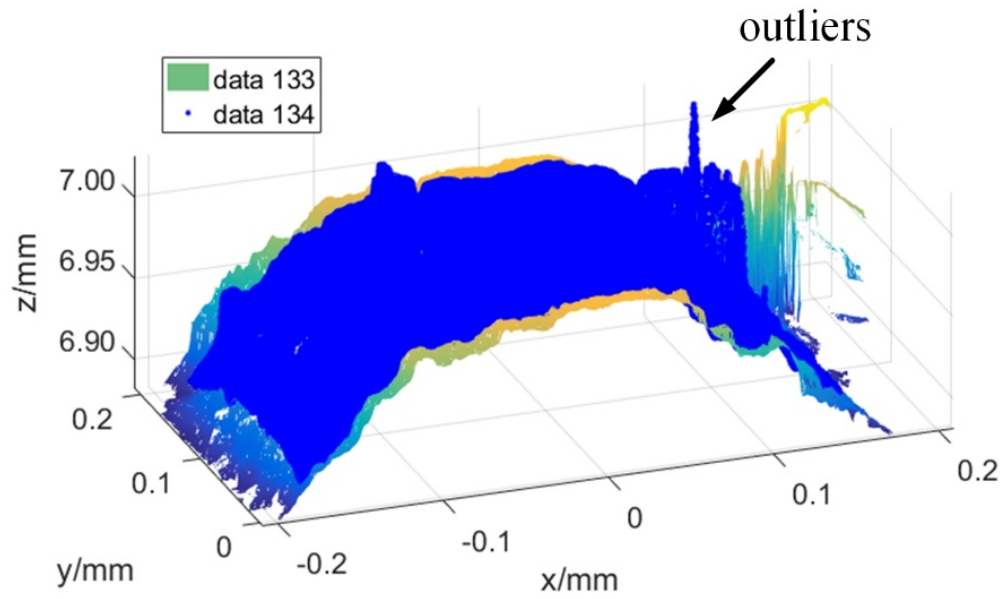

Figure 8 Registration between dataset 133 and 134 showing the outlier data point causing the large registration error.

After the adaptive registration process, the associated transformation matrices for all the original datasets were determined. The initial stitching was then completed, as shown in Figure 9. It was found that although the measurement error and registration error were very small (see Figure 7), the accumulated error along a chain of 200 datasets has resulted in a misalignment of approximately $0.5 \mathrm{~mm}$ in the perimeter direction between the 200th and first dataset, as shown in Figure 9(b). After self-calibration, the error was successfully compensated for, as shown in Figure 10. As a result, holistic measurement of both the geometrical form and surface topography of the grinding wheel is achieved. 


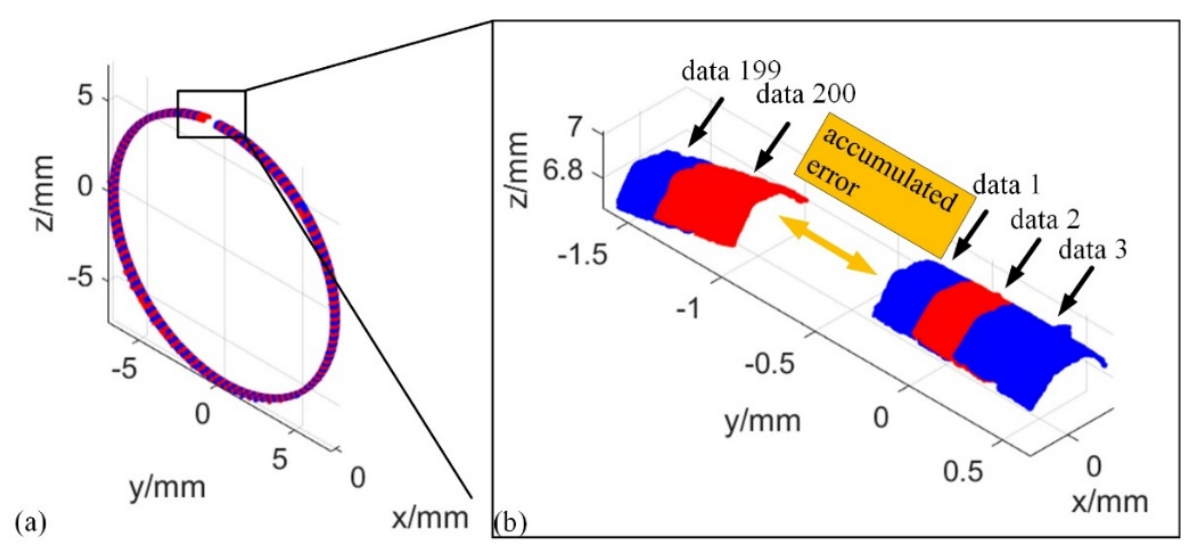

Figure 9 Initial stitching result, (a) with 200 datasets, (b) zoom-in view showing the accumulated error.

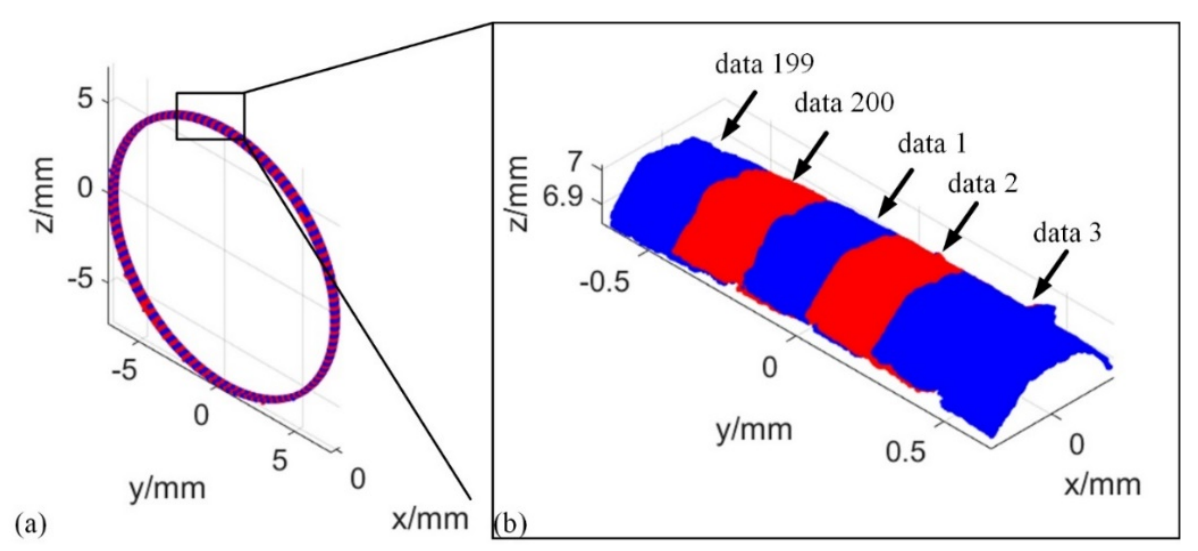

Figure 10 Stitching result after self-calibration, (a) with 200 datasets, (b) zoom-in view showing the accumulated error was corrected.

After the stitched datasets were corrected, the axis of the wheel was fitted using a least-squares method. The stitched surface is shown in Figure 11 and the colour bar represents the deviations from the best-fit cylindrical surface. A zoom-in view in Figure 11(b) shows the detailed surface topography of the grinding wheel and the colour bar represents the height in the $\mathrm{z}$ direction. The advantage of the proposed stitching method is that it not only obtains the geometrical form of the entire grinding wheel but also maintains high lateral resolution along the periphery direction, which is essential for characterising the surface texture of the grinding wheel. Such holistic measurement of the grinding wheel can benefit the study of surface generation in precision grinding [24].

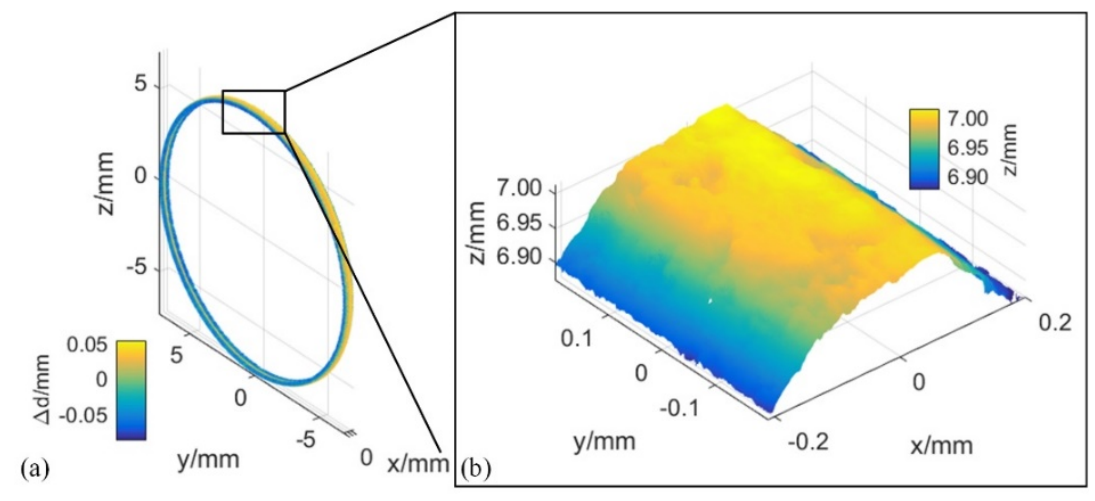

Figure 11 Stitching result, (a) holistic data with colour bar representing the deviation from fitted cylinder surface, (b) zoom-in view showing the surface topography of the grinding wheel with colour bar representing the $z$ height value. 
The same grinding wheel was also measured using a commercial FVM (Alicona G5) with a precision rotational axis (Real3D rotation stage). The resulting measurement is used as a reference to compare with the stitched revolving surface obtained with and without the SCRS method, as shown in Figure 12. The diameter of the grinding wheel determined for each obtained revolving surface is $14.2498 \mathrm{~mm}, 14.2440 \mathrm{~mm}$, and $13.9432 \mathrm{~mm}$, respectively. Since the top surface of the grinding wheel is not a flat surface, the diameters were calculated using the data in the range of $\left[R_{M A X}-0.08, R_{M A X}\right] \mathrm{mm}$, where $R_{M A X}$ is the maximum radius for the data determined with the outliers removed by a statistical outlier removal method [25]. The diameter of the grinding wheel was also measured using a Werth VideoCheck UA multisensor CMM with an optical probe and the result is $13.9795 \mathrm{~mm}$, where the measurement uncertainty of the CMM was estimated to be $0.83 \mu \mathrm{m}(\mathrm{k}=1)$. The results show that the determined diameter after selfcalibration is closer to the reference result from the CMM. The determined diameters are also summarised in Table 1. The accuracy of the proposed SCRS method is approximately $2 \%$ (by comparing the diameters) while the error may come from the measurement uncertainty caused by the surface roughness and local surface gradient [18, 19, 26, 27]. As discussed elsewhere [18], the measurement uncertainty of a CSI increases with increasing surface roughness and local surface gradient due to the fact that the height difference within one pixel of the CCD of the CSI is not distinguished. The height difference is larger when the surface roughness and the local surface gradient is larger, and vice versa. To further improve the accuracy, a new method should be developed to compensate the error by systematically modifying the measurement topography data and this will be studied in the future.

(a)

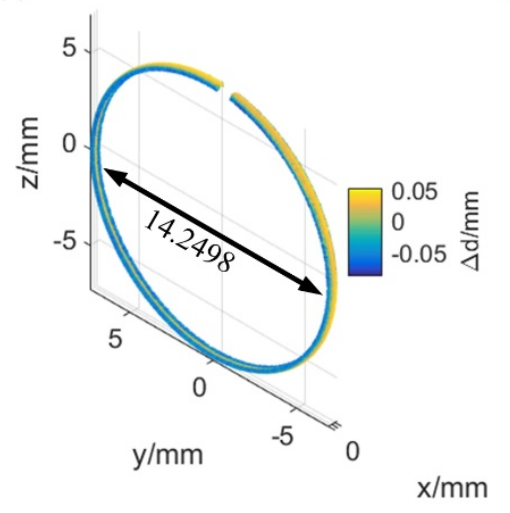

(b)

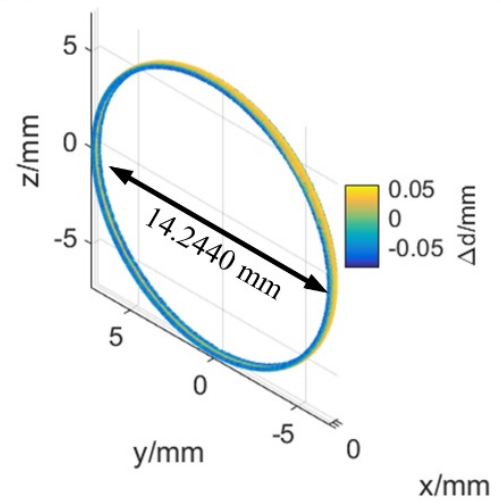

(c)

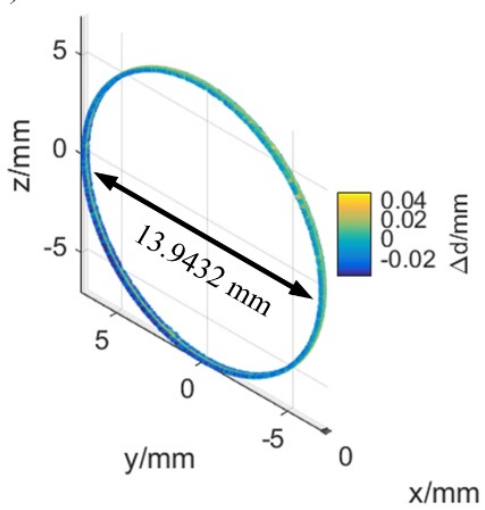

Figure 12 Stitching results, (a) initial stitching result, (b) with self-calibration, and (c) from FVM.

Table 1 Grinding wheel diameter determined after initial stitching, with self-calibration, from FVM and from CMM

\begin{tabular}{|l|c|c|c|c|}
\hline & Initial stitching & With self-calibration & From FVM & From CMM \\
\hline Determined diameter $(\mathrm{mm})$ & 14.2498 & 14.2440 & 13.9432 & 13.9795 \\
\hline
\end{tabular}

Another grinding wheel with a larger nominal diameter of $20 \mathrm{~mm}$ was measured with the proposed SCRS method. The surface roughness $\left(S_{a}\right)$ of the grinding wheel is approximately $1.3 \mu \mathrm{m}$. The number of measurements were also set to be 200 . With the larger grinding wheel, the overlapped region was reduced to approximately $30 \%$, but it was still larger than the suggested 20\% value to ensure the accuracy. The results are shown in Figure 13 . The estimated diameters for initial stitching, after self-calibration, from FVM and from CMM are $20.2534 \mathrm{~mm}, 20.2490$ $\mathrm{mm}, 19.8438 \mathrm{~mm}$ and $19.9157 \mathrm{~mm}$, respectively. The large gap in the data after initial stitching has been compensated using the self-calibration method. Diameter after self-calibration is closer to the reference data from the CMM, although there is still significant difference which may be caused by surface roughness and local surface gradient [18, 19, 26, 27], which will be investigated in the future to further improve the accuracy. The determined diameters are also summarised in Table 2. 

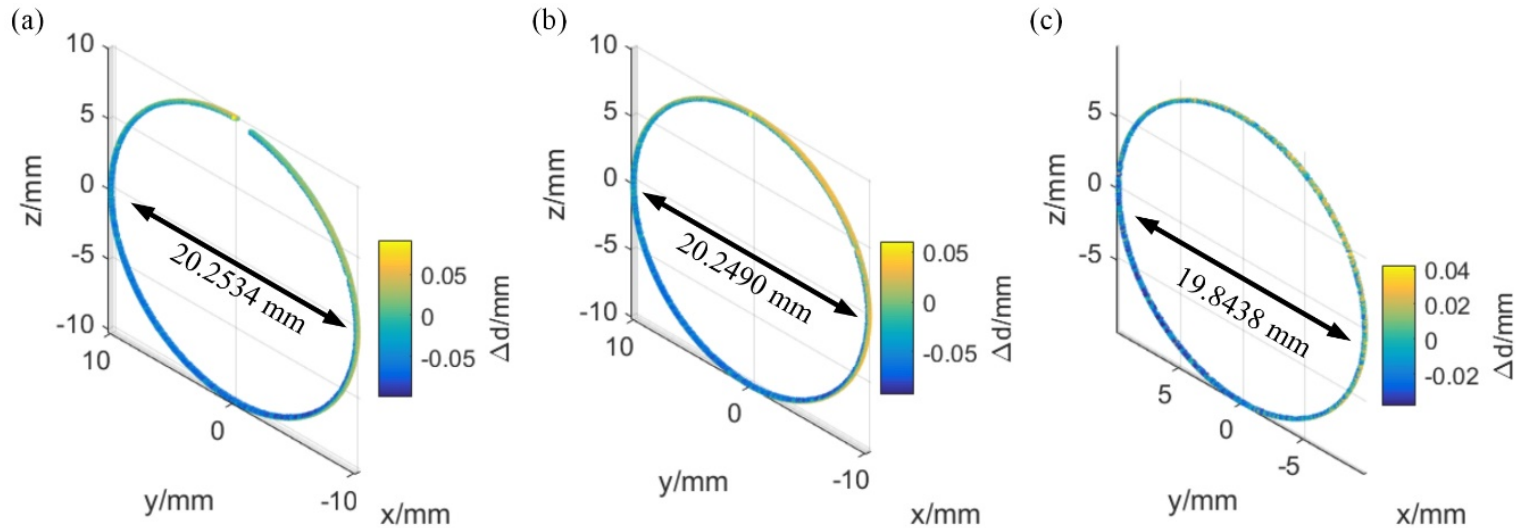

Figure 13 Stitching results for a grinding wheel with a nominal diameter of $20 \mathrm{~mm}$, (a) initial stitching result, (b) with self-calibration, and (c) from FVM.

Table 2 Grinding wheel diameter determined after initial stitching, with self-calibration, from FVM and from CMM

\begin{tabular}{|l|c|c|c|c|}
\hline & Initial stitching & With self-calibration & From FVM & From CMM \\
\hline Determined diameter $(\mathrm{mm})$ & 20.2534 & 20.2490 & 19.8438 & 19.9157 \\
\hline
\end{tabular}

\section{Conclusion}

A self-calibration rotational stitching (SCRS) method for measuring revolving surfaces is proposed in this paper. The proposed method utilises a rotational axis to rotate the revolving surface under a 2.5D surface measuring instrument for measurement of the entire revolving surface; and the sub-surfaces are stitched together using an adaptive registration method and a self-calibration method. With these methods, the accumulated stitching error is minimised with the help of the data itself. The proposed SCRS method enables measurement of both geometrical form and the surface topography of the revolving surface in one measurement session. Measurement accuracy is ensured by the stitching algorithm, adaptive registration and self-calibration. This is a novel method which is suitable for full 3D measurement of revolving surfaces. Future work is to further improve the accuracy by compensating the measurement error in the CSI caused by surface gradient and to implement this method to other 2.5D surface measuring instruments.

\section{Acknowledgements}

The work was mainly supported by the National Natural Science Foundation of China (No. 51675456). The authors also express their sincerely thanks to the European Metrology programme for innovation and research (EMPIR) from European Commission under the project no.: 15SIB01 FreeFORM. The authors also thank Mr. Shanshan Chen for providing the grinding wheel for the experiment, Mr. David Lai, Mr. Tsz Hin Fan for their help on the experiment setup and Dr Wenguang Hou, Dr Zuoping Chen for the valuable discussions on the mathematics of the stitching algorithm.

\section{References}

[1] Kong LB, Cheung CF, Lee WB. A theoretical and experimental investigation of orthogonal slow tool servo machining of wavy microstructured patterns on precision rollers. Precision Engineering. 2016;43:315-27. [2] Altintas Y, Woronko A. A piezo tool actuator for precision turning of hardened shafts. CIRP AnnalsManufacturing Technology. 2002;51:303-6.

[3] Inasaki I. Grinding Process Simulation Based on the Wheel Topography Measurement. CIRP Annals Manufacturing Technology. 1996;45:347-50. 
[4] Yang S-Y, Cheng F-S, Xu S-W, Huang P-H, Huang T-C. Fabrication of microlens arrays using UV microstamping with soft roller and gas-pressurized platform. Microelectronic engineering. 2008;85:603-9.

[5] Butler D, Blunt L, See B, Webster J, Stout K. The characterisation of grinding wheels using 3D surface measurement techniques. Journal of Materials Processing Technology. 2002;127:234-7.

[6] Oh JS, Song CK, Hwang J, Shim JY, Park CH. An ultra-precision lathe for large-area micro-structured roll molds. Journal of the Korean Society for Precision Engineering. 2013;30:1303-12.

[7] Wiegmann A, Stavridis M, Walzel M, Siewert F, Zeschke T, Schulz M, et al. Accuracy evaluation for subaperture interferometry measurements of a synchrotron mirror using virtual experiments. Precision engineering. 2011;35:183-90.

[8] Liu MY, Cheung CF, Cheng CH, Su R, Leach RK. A Gaussian process and image registration based stitching method for high dynamic range measurement of precision surfaces. Precision Engineering 2017;50:99-106.

[9] Fleig J, Dumas P, Murphy PE, Forbes GW. An automated subaperture stitching interferometer workstation for spherical and aspherical surfaces. Optical Science and Technology, SPIE's 48th Annual Meeting: International Society for Optics and Photonics, 2003. p. 296-307.

[10] Chen S, Li S, Dai Y, Zheng Z. Iterative algorithm for subaperture stitching test with spherical interferometers. JOSA A. 2006;23:1219-26.

[11] Peng J, Yu Y, Chen D, Guo H, Zhong J, Chen M. Stitching interferometry of full cylinder by use of the firstorder approximation of cylindrical coordinate transformation. Opt Express. 2017;25:3092-103.

[12] Peng J, Chen D, Yu Y. Stitching interferometry of a full cylinder without using overlap areas. Meas Sci Technol. 2017;28:085001.

[13] Weckenmann A, Bruning J, Patterson S, Knight P, Bryan J. Grazing incidence interferometry for high precision measurements of cylindrical form deviations. CIRP Annals-Manufacturing Technology. 2001;50:381-4.

[14] Guo H, Chen M. Multiview connection technique for 360-deg three-dimensional measurement. Optical Engineering. 2003;42:900-5.

[15] Vissiere A, Nouira H, Damak M, Gibaru O, David J-M. A newly conceived cylinder measuring machine and methods that eliminate the spindle errors. Measurement Science and Technology. 2012;23:094015.

[16] Neugebauer M, Lüdicke F, Bastam D, Bosse H, Reimann H, Töpperwien C. A new comparator for measurement of diameter and form of cylinders, spheres and cubes under clean-room conditions. Measurement Science and Technology. 1997;8:849.

[17] Besl PJ, McKay ND. A method for registration of 3-D shapes. IEEE Transactions on pattern analysis and machine intelligence. 1992;14:239-56.

[18] Liu MY, Cheung CF, Ren MJ, Cheng CH. Estimation of measurement uncertainty caused by surface gradient for a white light interferometer. Applied Optics. 2015;54:8670-7.

[19] Su R, Wang Y, Coupland J, Leach R. On tilt and curvature dependent errors and the calibration of coherence scanning interferometry. Opt Express. 2017;25:3297-310.

[20] Ye S-w, Yang P, Peng Y-f. A profile measurement method of large aspheric optical surface based on optimal stitching planning. Precision Engineering. 2016;45:90-7.

[21] Gao F, Coupland J, Petzing J. V-groove measurements using white light interferometry. Photon06.

Manchester2006. p. 4-7.

[22] Leach R, Smith ST. Basics of Precision Engineering: CRC Press, 2018.

[23] Moré JJ. The Levenberg-Marquardt algorithm: Implementation and theory. In: Watson GA, editor. Numerical Analysis: Proceedings of the Biennial Conference Held at Dundee, June 28-July 1, 1977. Berlin, Heidelberg:

Springer Berlin Heidelberg, 1978. p. 105-16.

[24] Chen S, Cheung C, Zhao C, Zhang F. Simulated and measured surface roughness in high-speed grinding of

silicon carbide wafers. The International Journal of Advanced Manufacturing Technology. 2016;91:719-30.

[25] Rusu RB, Cousins S. 3D is here: Point Cloud Library (PCL). 2011 IEEE International Conference on Robotics and Automation2011. p. 1-4.

[26] Gao F, Leach RK, Petzing J, Coupland JM. Surface measurement errors using commercial scanning white light interferometers. Measurement Science and Technology. 2008;19:015303.

[27] Pavliček P, Hýbl O. White-light interferometry on rough surfaces--measurement uncertainty caused by surface roughness. Applied optics. 2008;47:2941-9. 